Opinion

\section{Laser spears for the Russian army}

\section{Victor V Apollonov*}

A.M. Prokhorov GPI RAS, Vavilov str. 38, 119991, Moscow, Russia

\section{Abstract}

The paper is devoted to the problem of high-energy solid-state laser arms, demonstrating the highest efficiency in the terms of weight and sizing. We can predict a very bright future for such laser systems as a significant means of defeating the enemy. The problem of scalability for such an approach based on fiber laser technology is the key question of our days for many companies of the world. At the same time, it is clear that a tactical LW based on solid-state technology with a weight factor of $5 \mathrm{~kg} / \mathrm{kW}$ with the total weight of a laser complex significantly less than a ton can be created. But the level of output power around $500 \mathrm{~kW}$ is very close to the limit for the fiber technology...

The creation of high-tech weapons is possible only on the basis of solid-state technologies

Laser weapons are firmly established in real life and their first use in the war of Argentina and England for the Falkland Islands, when the simplest LW was used to blind the enemy, was just the beginning. The development and creation of inefficient and very expensive laser monsters on gas-dynamic and chemical bases in the United States and on electric-discharge, gas-dynamic and chemical bases in the USSR became the next stage in the development of the laser weapons epic. Then there were spectacular, but not effective, LW demonstrations at the Farnborough International Air show and for the fight against drones in the Persian Gulf. It is clear that in order for lasers to become a serious weapon, it is necessary to significantly reduce the size of the complexes, increase their efficiency and increase the output energy. Laser "toys" with a power of up to 60 kilowatts in a dense and humid air environment for use in power mode are ineffective. But even at the Giga / terawatt level, pulses with the nano/ micro-joule energy of a single ultrashort pulse impact will not be a panacea. Both the energy sector and its peak capacity are important. The mechanisms of the functional effect of LW are well studied, there is a niche for its application, and the transition to the creation of a high-frequency pulse-periodic (P-P) mode is not accidental. And yet these are only cautious attempts to understand what to work with. Research in the field of combat lasers is already at the level wherein the fairly near future it is possible to create a significant means of defeating the enemy [1].

\section{The path is long and expensive}

Strategic and tactical LW - a new paradigm for the
More Information

*Address for Correspondence:

Acad. Victor V Apollonov, A.M. Prokhorov GPI RAS, Vavilov str. 38, 119991, Moscow, Russia, Email: vapollo@rambler.ru

Submitted: December 13, 2021

Approved: February 07, 2022

Published: February 08, 2022

How to cite this article: Apollonov VV.

Laser spears for the Russian army. Int J Phys Res Appl. 2022; 5: 007-010.

DOI: 10.29328/journal.ijpra.1001043

Copyright: ๑ 2022 Apollonov VV. This is an open access article distributed under the Creative Commons Attribution License, which permits unrestricted use, distribution, and reproduction in any medium, provided the original work is properly cited.

Check for updates

OPEN ACCESS

development of weapons, tactics, and strategies for their use. It acts instantly and in a straight line, does not require the calculation of ballistics. This means that in the upper atmosphere, and even more so in space, where there is practically no absorption and scattering of radiation, lasers will determine a lot, if not everything, in the strategy and tactics of armed struggle. The world will begin to learn about this at the end of 2022 when the United States will partially equip military aircraft (and not only) with a compact and light tactical LW, capable of operating in power and functional modes for tens and hundreds of kilometers. At the next stage, when strategic lasers are launched into space, and this will inevitably happen through the efforts of the Pentagon, the true price of the new weapon will become well understood.

In this regard, the American program "Self - defense based on a high - energy laser" - SHiELD (Self-protect High Energy Laser Demonstrator) is of interest) about the creation of a fifth-or sixth-generation fighter with a laser onboard for defense against air-to-air and surface-to-air missiles. For the company, Northrop Grumman-the design of the laser beam control system, and the development of the laser itself went to Lockheed Martin. Boeing has been tasked with integrating the entire weapon system into a single SHiELD complex. The program was developed in stages: on the basis of fiber lasers with the spectral addition of radiation from single sources, solid-state systems with an average power of 30 kilowatts were created, then 60 and even 150. The choice of a fiber device as a single element of power addition is determined by its high radiation quality, compactness, and low weight characteristics. Today, the weight factor is increased to $5 \mathrm{~kg}$ 
/ $\mathrm{kW}$, including the weight of the laser, the power supply, the excess heat removal system, and the platform that carries the complex as a whole. Lockheed Martin plans to increase this factor to $2 \mathrm{~kg} / \mathrm{kW}$, which means that the 10-megawatt LW complex will weigh only 20 tons. This is promising for the development of strategic laser weapons. The technology of the created prototype system of 300 kilowatts with a total weight of only 600 kilograms has already been developed. For comparison, I note that in gas, chemical, and steam-based lasers, which have already been abandoned in the United States (COIL, GDL, HF/DF, alkali metal vapors), the weight factor is in the range of $200-400 \mathrm{~kg} / \mathrm{kW}$, which means that a megawatt LW complex will hardly fit in a heavy aircraft carrier. And to achieve air supremacy, it is necessary to equip serial combat aircraft with light and compact tactical air defense systems weighing within a few hundred kilograms. The weight factor is of particular importance when equipping spacecraft with lasers, and this issue is already on the Pentagon's agenda. It is necessary to create laser complexes with an output power of several tens of megawatts. Therefore, the LW on the basis of advanced solid-state technology allows us to look to the future with confidence and we can talk about the creation of the entire complex of laser weapons that meet the requirements of the Russian Armed Forces. Nevertheless, the technology of adding up the radiation of fiber lasers for a number of reasons is suitable only for tactical LW with a power of up to 500 kilowatts.

\section{Difficulties of addition}

There is another solid-state laser disk. The idea of Academician Nikolai Basov is already 55 years old, but it seems that this rule of building high-power laser systems will be dominant for a long time. With the same very favorable weight factor of less than $5 \mathrm{~kg} / \mathrm{kW}$, this design principle allows the implementation of a high-energy high-frequency P-P mode, since the aperture of an existing disk laser has a diameter of about 1.5 centimeters, which is significantly larger than the diameter of the active body of a fiber laser. To increase the average power of the system, several disks are added to the optical sequence ZIG-ZAG, the value of the average power of such a module is already 50 kilowatts. Modules, as in the case of fiber systems, can be built in parallel, and the power is added to the target. Based on these figures, it can be seen that a 100 kilowatt laser will weigh less than 500 kilograms! The parallel addition of the modules leads to an increase in the total aperture and, consequently, to the possibility of increasing the pulse energy in a high-frequency periodic sequence, which qualitatively changes the interaction mechanism, allowing us to obtain new effects on the target. It should be noted that the LW complexes of significantly higher average power are needed to perform the tasks of the Armed Forces of the Russian Federation. But from the disk geometry of modules with a capacity of even 75 kilowatts (this increase is planned due to the quality of reflective coatings) to the power level of the entire system of about 10 megawatts - a giant distance. It is not possible to combine the power of more than a hundred modules in a single beam in a mobile complex.

\section{How to zoom in}

Obviously, we need a different design scheme for a solidstate complex, which allows scaling its average power while maintaining the weight factor. Much is already clear today. First of all, it is impossible to create light laser systems based on chemical, gas, and steam bases. The technology of tactical complexes already worked out in the United States is based on a fiber type of laser, the research of which began in Russia, later they were developed and patented in the United States by an emigrant from the Russian Federation. Now fiber devices are produced in the United States, Germany, and Russia. We copy the technology of creating tactical LW and the technology of spectral addition of fiber laser radiation, while using imported element base for a small level of medium power, selectively allowed by the US State Department for export. On this basis, it is impossible to fully equip the Russian Armed Forces with high-energy tactical complexes. Fiber high-energy lasers, due to physical limitations, cannot be high-frequency P-P with large average power, and the spectral composition of the radiation of compact and light weapons systems is limited by the tactical power level. This is the limitation of this technology: not only the strategic level of power but also many new effective applications based on it are physically unattainable. The core geometry of the active element of the laser, as well as the" slab" (in the form of a plate) geometry, also does not allow to overcome the tactical level of the final laser complex based on solid-state technology. At the same time, these geometries of the active element of the laser lead to significant weight and dimensions of the device with a low level of reliability.

\section{The basis of the combat laser is the module}

The only promising approach to creating a line of solidstate complexes from the tactical to the strategic level today is the mono-module technology proposed in Russia. It is obvious that the new aviation laser product under the SHiELD program will be able not only to protect against a missile attack but also to become a threat to enemy military equipment and aircraft. Laser radiation is significantly absorbed and dispersed in desert conditions, where many tests of tactical complexes for combating drones have been conducted. It is known that the power of the beam falls three times at each subsequent mile of the distance. And this complicates the use of lasers at high humidity. But it does not follow that the law is inefficient in principle. It is necessary to increase the power of the source and go to a higher level of average power, to other time modes of the generated radiation. In addition to destroying drones, there are other military tasks - for example, the use of lasers in the upper atmosphere. At altitudes of seven to nine kilometers, the environment is more transparent and the target ranges for destroying aircraft, even for a power level of 100-150 kilowatts, can reach tens of kilometers. And if we are 
talking about megawatt tessituras, then scientists from the United States and the USSR experimentally proved the reality of the range of active operation of the LW up to one hundred kilometers. Another thing is that all this is a tactical range and it is necessary to increase the average power of the LW to achieve a strategic level of a thousand kilometers or more with the strictest control of the weight and size of the device. Hence the conclusion: it is the fiber and disk geometries of the active element of the laser that determine the future of the LW for almost all branches of the armed forces.

\section{Space as a battlefield}

Let's think about the space tasks of combat lasers. There is practically no air and water vapor in the orbit, which means that there is no absorption and scattering of radiation and fluctuations in the density of the medium. In this case, the usual adaptive optics are not needed. Only the optical quality of the generated radiation will be required, and hence the quality of static power optics made of silicon carbide, which was first implemented for this purpose up to a meter-sized mirror in Russia. However, difficulties are added, such as the release of excess heat (the efficiency of the laser is far from 100 percent) and the accuracy of targeting at strategic ranges. Next, a largescale laser complex of megawatt power must be managed to put into space. Americans focus on solid-state technology, which allows you to provide a weight factor of LW at or less than $5 \mathrm{~kg} / \mathrm{kW}$. And here, the combat complex "Peresvet", which was not created using the solid-state technology of the working body of the laser, according to the Russian experts will have difficulties in maintaining the optimal weight and dimensions of the final system in the further development of the project. Recall that the technologies of chemical, gas, and alkali metal vapor lasers rejected by the Americans do not provide the implementation of the weight factor at a level of less than $5 \mathrm{~kg} / \mathrm{kW}$, since it goes into the range of values of 200 $400 \mathrm{~kg} / \mathrm{kW}$. This means that the strategic laser system, built on outdated physical principles, is so heavy and cumbersome that it is really only suitable for use on earth. We must also keep in mind the task of the first stage set by the Joint Chiefs of Staff US, which refers to the installation of tactical com-plexes with a capacity of 100-150 kilowatts on the US aircraft by the end of 2022. Such an LW based on solid-state technology with a weight factor of $5 \mathrm{~kg} / \mathrm{kW}$ will weigh significantly less than a ton. At the same time, lasers with a power of 100 kilowatts on the old physical principles would weigh about 20 tons. And this, if we evaluate it very optimistically. It is clear that with such complexes, it is impossible to win the fight for air, and at the second stage for space.

\section{Wounded - not killed}

The functional defeat of the target is said when, due to the limited energy reserve, it is impossible to provide the required power density for a power defeat. It is necessary to strive for the creation of an LW that ensures the guaranteed destruction of enemy equipment. For example, an impulse cuts off the wing of an airplane or a cruise missile (CM), and they cease to function. With significantly lower energy, only functional damage is possible. For example, the rocket's laser damaged the guidance system. But it continues to fly, and in this case, there is no certainty that the CM will not be able to destroy the object of attack. Thus, the functional defeat of the target is good when power is objectively impossible with a lack of average laser radiation power at a given distance. And this means that the developers of the LW need to think about light and compact strategic weapons that can destroy objects in the power mode, that is, always have a reserve of energy in the beam. It should be noted that not all solid-state technologies are equally effective. The core geometry of the active element in relation to the large average capacities of complexes is finally forgotten. The geometry of the "slab" is significantly limited by the output power level of a single module. It is about the same with fiber technology. A further increase in the average power of the LW is then possible due to the spectral addition of the radiation of individual modules. Simple summation of radiations (purely geometric) is inefficient for a number of reasons. Attempts at spectral addition of "fiber" in the United States led to the creation of a prototype LW with an average power of 300 kilowatts. The limitations of this approach in terms of further scaling of the average power are convincingly shown (500 kW, not more). The only correct direction for solid-state technology is to obtain a single laser beam of radiation in a single resonator with a large-diameter disk active element. In the approach we developed in 2013 to solve the scaling problem, it is possible to increase the average power of the LW complex to tens of megawatts of average power. In the high-frequency P-P mode, the peak power of the pulse sequence can be increased by orders of magnitude. And this is exactly the goal that the creators of LW have been striving for and are striving for all over the world. And only in this way can the problems of the Armed Forces of the Russian Defense Ministry be solved both in power and in functional modes. Simultaneously with the increase in the energy contribution to the target in the highfrequency P-P mode, the impact on the target is additionally achieved by a number of factors: a wide spectrum of the light field, electromagnetic radiation, giant currents, and the strongest sound field (up to 20 percent of the laser energy can go into sound). Thus, the possibilities of complex impact on targets are significantly expanded. At the same time, all these factors occur on the target surface, and the radiation is delivered to the object at a small size in comparison with the existing traditional EW telescope (antenna).

\section{Monopoly harms competition}

In the Russian Federation, there is a monopoly on the creation of tactical and strategic LW, and as you know, monopolists do not tolerate competition. For comparison: in the United States, Lockheed Martin, Textron, Northrop Grumman, Boeing, Raytheon, General Atomics, and other companies are working on the creation of high-energy 
weapons. We also need to create at least one more center that deals with fiber-based lasers, as well as on the basis of monomodular disk geometry and high-frequency P-P generation mode. Unjustifiably little attention is paid to the development of the element base of promising types of solid-state weapons, which, when creating strategic, and therefore the entire line of tactical lasers based on mono module disk geometry, will not quickly equip the Russian army with modern and promising high-tech weapons.

\section{Conclusion}

Thus, based on what has been written, several conclusions can be drawn:

1. Solid-state LW complexes based on fiber technology have already been created in many countries of the world, the US is in particular.

2. The chosen way to increase the output power of the complex - spectral addition - is an effective method, but, unfortunately, it is limited by the tactical level of output power, not more than $500 \mathrm{~kW}$ for mobile systems with a weight factor of $5 \mathrm{~kg} / \mathrm{kW}$.

3. The high-frequency pulse repetition mode of a highenergy laser seems to be a powerful tool for influencing the target in the "smart interaction mode" (functional impact).

4. The only true way to move from a functional impact (limited average power) to a power one (many megawatts of average power) is the implementation of the proposed by Academician N.G. Basov disk-type laser, but in its monomodular version, provided that the problem of amplified spontaneous emission is overcome.

\section{References}

1. VPK kurier № 11(874). 2021 